\title{
Recurrence Relations for Moments of Generalized Order Statistics for Inverse Weibull Distribution and Some Characterizations
}

\author{
Saman Hanif Shahbaz \\ Department of Statistics, Faculty of Science, King Abdulaziz University \\ Jeddah Saudi Arabia \\ saman.shahbaz17@gmail.com \\ Mohammad Ahsanullah \\ Department of Management Sciences, Rider University \\ Lawrenceville, USA \\ ahsan@rider.edu \\ Muhammad Qaiser Shahabaz \\ Department of Statistics, Faculty of Science, King Abdulaziz University \\ Jeddah Saudi Arabia \\ qshahbaz@gmail.com
}

Received 3 January 2016

Accepted 25 October 2016

\begin{abstract}
We present recurrence relations for single, inverse, product and ratio moments of Generalized Order Statistics when sample is available from an Inverse Weibull Distribution. These relations enable computation of higher order moments using corresponding lower order moments. Some Characterizations of the distribution are also given in terms of conditional moments.
\end{abstract}

Keywords: Recurrence Relations; Inverse Weibull Distribution; Characterizations.

2000 Mathematics Subject Classification: 22E46, 53C35, 57S20

\section{Introduction}

Recurrence relations for moments of order statistics has been an old topic of study. Several identities have been derived which relate higher order moments of order statistics with corresponding lower order moments, see for example Cole [11], Arnold et al. [1] and David and Nagaraja [12]. Since emergence of record statistics by Chandler [10], various authors have studied distributions of records. Various authors have studied recurrence relations between moments of records for certain probability distributions, see for example Ahsanullah [2], Balakrishnan and Ahsanullah [6], Balakrishnan et al. [7], Bieniek and Szynal [9], Pawlas and Szynal [17] among others. A comprehensive review of record statistics can be found in Ahsanullah [3]. 
Kamps [13] has introduced generalized order statistics (gos) as a unified model for ordered random variables. The gos are defined as under:

Suppose a random sample of size $n$ is available from the distribution $F(x)$ then the quantities $X_{r: n, \tilde{m}, k}$ are the Generalized Order Statistics (GOS) from the distribution $F(x)$, if their joint density function is of the form

$$
\begin{aligned}
f_{1, \ldots, n: n, \tilde{m}, k}\left(x_{1}, \ldots, x_{n}\right)= & k\left(\prod_{j=1}^{n-1} \gamma_{j}\right)\left\{1-F\left(x_{n}\right)\right\}^{k-1} f\left(x_{n}\right) \\
& \times\left[\prod_{i=1}^{n-1}\left\{1-F\left(x_{i}\right)\right\}^{m_{i}} f\left(x_{i}\right)\right]
\end{aligned}
$$

where and is defined on the cone $F^{-1}(0)<x_{1} \leq x_{2} \leq \ldots \leq x_{n}<F^{-1}(1)$. If $m_{1}=$ $m_{2}=\cdots=m_{n-1}=m$, then GOS are denoted as $X_{r: n, m, k}$, where $r$ is a positive integer such that $1 \leq r \leq n$.

The marginal density function of $r$ th gos is given by Kamps [13] as:

$$
f_{r: n, m, k}(x)=\frac{C_{r-1}}{(r-1) !} f(x)\{1-F(x)\}^{\gamma_{r}-1} g_{m}^{r-1}[F(x)],
$$

where $C_{r-1}=\prod_{j=1}^{r} \gamma_{j}, \gamma_{j}=k+(n-j)(m+1)$ and

$$
\begin{aligned}
h_{m}(x) & =\left\{\begin{array}{cc}
-\frac{1}{m+1}(1-x)^{m+1} ; m \neq-1 \\
-\ln (1-x) \quad ; m=-1
\end{array} ; x \in[0,1)\right. \\
g_{m}(x) & =h_{m}(x)-h_{m}(0) \\
& =\left\{\begin{array}{cc}
\frac{1}{m+1}\left[1-(1-x)^{m+1}\right] & {[m \neq-1} \\
-\ln (1-x) ; & m=-1
\end{array} ; x \in[0,1) .\right.
\end{aligned}
$$

Further the joint density function of two $g o s X_{r: n, m, k}$ and $X_{s: n, m, k}$ for $r<s$ is given by Kamps [13] as

$$
\begin{aligned}
f_{r, s: n, m, k}\left(x_{1}, x_{2}\right) & =\frac{C_{s-1}}{(r-1) !(s-r-1) !} f\left(x_{1}\right) f\left(x_{2}\right) \\
& \times\left\{1-F\left(x_{1}\right)\right\}^{m} g_{m}^{r-1}\left\{F\left(x_{1}\right)\right\} \\
& \times\left\{1-F\left(x_{2}\right)\right\}^{\gamma_{s}-1}\left[h_{m}\left\{F\left(x_{2}\right)\right\}-h_{m}\left\{F\left(x_{1}\right)\right\}\right]^{s-r-1} .
\end{aligned}
$$

The gos reduces to order statistics for $m=0$ and $k=1$ and it reduces to record statistics for $m=-1$. Several authors have studied gos for various distributions in context of recurrence relations for moments of gos. Athar and Islam [4] have provided recurrence relations for moments of gos for a general class of distributions. Athar et al. [5] have studied recurrence relations for moments for gos for Marshall-Olkin extended Weibull distribution and have provided a characterization. Kumar [15] have studied recurrence relations for moments of Kumaraswamy distribution. Mohsin et al. [16] have provided recurrence relations for moments of gos for Rayleigh distribution.

The gos provide a unified model for random variables arranged in increasing order. Burkschat et al. [8] have introduced lower or dual generalized order (dgos) statistics as a unified model for 
random variables arranged in decreasing order of magnitude. Various authors have studied the recurrence relations for moments of $d g o s$ for certain distributions. Pawlas and Szynal [18] have obtained the recurrence relations for moments of dgos for Inverted Weibull distribution with density

$$
f(x)=\frac{\alpha \beta}{x^{\beta+1}} \exp \left(-\frac{\alpha}{x^{\beta}}\right) .
$$

Kotb et al. [14] have studied recurrence relations for moments of $d g o s$ for a general class of inverted distributions defined as

$$
f(x)=\frac{\theta \lambda^{\prime}(x)}{\lambda^{2}(x)} \exp \left\{-\frac{\theta}{\lambda(x)}\right\} ; a<x<b,
$$

where $\lambda(x)$ is some function of random variable $X$. The inverted class of distributions given in (5) provide Inverted Weibull distribution as a special case when $\lambda(x)=x^{\beta}$. Kotb et al. [14] have also shown that the recurrence relations for moments of dgos for Inverted Weibull distribution can be obtained from those of general class of inverted distributions.

It is of interest to note that the recurrence relations for moments of gos have not been studied in context of inverted distributions. In this paper we study the recurrence relations for moments of gos for Inverted Weibull distribution and provide certain special cases. These relations are studied in the following sections

\section{Relation for Single Moments}

Athar and Islam [4] have provided following general expression for relation between single moments of gos

$$
\mu_{r: n, m, k}^{p}-\mu_{r-1: n, m, k}^{p}=\frac{p C_{r-2}}{(r-1) !} \int_{-\infty}^{\infty} x^{p-1}\{1-F(x)\}^{\gamma_{r}} g_{m}^{r-1}[F(x)] d x,
$$

where $\mu_{r: n, m, k}^{p}=E\left(X_{r: n, m, k}^{p}\right)$ etc. It is to be observed that $p$ th moment of Inverted Weibull distribution exist when $\beta>p$. We will, therefore, obtain the recurrence relations for single moments when $\beta>p$. We will use expression (6) to obtain relation for single moments of gos for Inverted Weibull distribution. For this consider the density function of Inverted Weibull distribution given in (4). The distribution function corresponding to (4) is

$$
F(x)=\exp \left(-\frac{\alpha}{x^{\beta}}\right)
$$

It can be easily noted that following relation hold between density and distribution function

$$
1-F(x)=\left[\frac{x^{\beta+1}}{\alpha \beta}\left\{\exp \left(\frac{\alpha}{x^{\beta}}\right)-1\right\}\right] f(x) .
$$

We will use relation (7) to derive the recurrence relation for single moments of gos for Inverted Weibull distribution. For this consider (6) as

$$
\begin{aligned}
\mu_{r: n, m, k}^{p}-\mu_{r-1: n, m, k}^{p}= & \frac{p C_{r-2}}{(r-1) !} \int_{-\infty}^{\infty} x^{p-1}\{1-F(x)\} \\
& \times\{1-F(x)\}^{\gamma_{r}-1} g_{m}^{r-1}[F(x)] d x .
\end{aligned}
$$


Now using (7) in above equation we have

$$
\begin{aligned}
\mu_{r: n, m, k}^{p}-\mu_{r-1: n, m, k}^{p}= & \frac{p C_{r-2}}{(r-1) !} \int_{0}^{\infty} x^{p-1}\left[\frac{x^{\beta+1}}{\alpha \beta}\left\{\exp \left(\frac{\alpha}{x^{\beta}}\right)-1\right\}\right. \\
& \times f(x)]\{1-F(x)\}^{\gamma_{r}-1} g_{m}^{r-1}[F(x)] d x .
\end{aligned}
$$

or

$$
\begin{aligned}
\mu_{r: n, m, k}^{p}-\mu_{r-1: n, m, k}^{p}= & \frac{p C_{r-2}}{(r-1) !} \int_{0}^{\infty} x^{p-1} \frac{x^{\beta+1}}{\alpha \beta} \exp \left(\frac{\alpha}{x^{\beta}}\right) f(x) \\
& \times\{1-F(x)\}^{\gamma_{r}-1} g_{m}^{r-1}[F(x)] d x \\
& -\frac{p C_{r-2}}{(r-1) !} \int_{0}^{\infty} x^{p-1} \frac{x^{\beta+1}}{\alpha \beta} f(x) \\
& \times\{1-F(x)\}^{\gamma_{r}-1} g_{m}^{r-1}[F(x)] d x
\end{aligned}
$$

or

$$
\begin{aligned}
\mu_{r: n, m, k}^{p}-\mu_{r-1: n, m, k}^{p}= & \frac{p}{\alpha \beta \gamma_{r}} \sum_{j=0}^{\infty} \frac{\alpha^{j}}{j !} \frac{C_{r-1}}{(r-1) !} \int_{0}^{\infty} x^{p-\beta(j-1)} f(x) \\
& \times\{1-F(x)\}^{\gamma_{r}-1} g_{m}^{r-1}[F(x)] d x \\
& -\frac{p}{\alpha \beta \gamma_{r}} \frac{C_{r-1}}{(r-1) !} \int_{0}^{\infty} x^{p+\beta} f(x) \\
& \times\{1-F(x)\}^{\gamma_{r}-1} g_{m}^{r-1}[F(x)] d x
\end{aligned}
$$

or

$$
\mu_{r: n, m, k}^{p}-\mu_{r-1: n, m, k}^{p}=\frac{p}{\alpha \beta \gamma_{r}}\left\{\sum_{j=0}^{\infty} \frac{\alpha^{j}}{j !} \mu_{r: n, m, k}^{p-\beta(j-1)}-\mu_{r: n, m, k}^{p+\beta}\right\} .
$$

Remark 2.1. Using $m=0$ and $k=1$ in (8) we obtain recurrence relation for single moments of order statistics for Inverted Weibull distribution as

$$
\mu_{r: n}^{p}-\mu_{r-1: n}^{p}=\frac{p}{\alpha \beta(n+r-1)}\left\{\sum_{j=0}^{\infty} \frac{\alpha^{j}}{j !} \mu_{r: n}^{p-\beta(j-1)}-\mu_{r: n}^{p+\beta}\right\} .
$$

Remark 2.2. Using $m=-1$ in (8) we obtain recurrence relation for single moments of $K$-record values for Inverted Weibull distribution as

$$
\mu_{K(r)}^{p}-\mu_{K(r-1)}^{p}=\frac{p}{\alpha \beta k}\left\{\sum_{j=0}^{\infty} \frac{\alpha^{j}}{j !} \mu_{K(r)}^{p-\beta(j-1)}-\mu_{K(r)}^{p+\beta}\right\} .
$$

\section{Relation for Inverse Moments}

In this section we derive recurrence relation for inverse moments of gos. The inverse moments of gos are defined as

$$
\mu_{r: n, m, k}^{-p}=E\left(X_{r: n, m, k}^{-p}\right)=\int_{-\infty}^{\infty} x^{-p} f_{r: n, m, k}(x) d x .
$$


We consider following relation for inverse moments of gos

$$
\mu_{r: n, m, k}^{-p}-\mu_{r-1: n, m, k}^{-p}=-\frac{p C_{r-2}}{(r-1) !} \int_{-\infty}^{\infty} x^{-p-1}\{1-F(x)\}^{\gamma_{r}} g_{m}^{r-1}[F(x)] d x,
$$

or

$$
\begin{aligned}
\mu_{r: n, m, k}^{-p}-\mu_{r-1: n, m, k}^{-p}= & -\frac{p C_{r-2}}{(r-1) !} \int_{-\infty}^{\infty} x^{-p-1}\{1-F(x)\}^{\gamma_{r}-1} \\
& \times\{1-F(x)\} g_{m}^{r-1}[F(x)] d x .
\end{aligned}
$$

Now using (7) in (11) we have

$$
\begin{aligned}
\mu_{r: n, m, k}^{-p}-\mu_{r-1: n, m, k}^{-p}= & -\frac{p C_{r-2}}{(r-1) !} \int_{0}^{\infty} x^{-p-1}\left[\frac{x^{\beta+1}}{\alpha \beta}\left\{\exp \left(\frac{\alpha}{x^{\beta}}\right)-1\right\}\right. \\
& \times f(x)]\{1-F(x)\}^{\gamma_{r}-1} g_{m}^{r-1}[F(x)] d x .
\end{aligned}
$$

or

$$
\begin{aligned}
\mu_{r: n, m, k}^{-p}-\mu_{r-1: n, m, k}^{-p}= & -\frac{p}{\alpha \beta \gamma_{r}} \sum_{j=0}^{\infty} \frac{\alpha^{j}}{j !} \frac{C_{r-1}}{(r-1) !} \int_{0}^{\infty} x^{-p-\beta(j-1)} f(x) \\
& \times\{1-F(x)\}^{\gamma_{r}-1} g_{m}^{r-1}[F(x)] d x \\
& +\frac{p}{\alpha \beta \gamma_{r}} \frac{C_{r-1}}{(r-1) !} \int_{0}^{\infty} x^{-p+\beta} f(x) \\
& \times\{1-F(x)\}^{\gamma_{r}-1} g_{m}^{r-1}[F(x)] d x
\end{aligned}
$$

or

$$
\mu_{r: n, m, k}^{-p}-\mu_{r-1: n, m, k}^{-p}=\frac{p}{\alpha \beta \gamma_{r}}\left\{\mu_{r: n, m, k}^{-p+\beta}-\sum_{j=0}^{\infty} \frac{\alpha^{j}}{j !} \mu_{r: n, m, k}^{-p-\beta(j-1)}\right\} .
$$

Remark 3.1. Using $\beta=2$ in (12) we have following recurrence relations for inverse moments of gos for Inverted Rayleigh distribution

$$
\mu_{r: n, m, k}^{-p}-\mu_{r-1: n, m, k}^{-p}=\frac{p}{2 \alpha \gamma_{r}}\left\{\mu_{r: n, m, k}^{-p+2}-\sum_{j=0}^{\infty} \frac{\alpha^{j}}{j !} \mu_{r: n, m, k}^{-p-2 j+2}\right\} .
$$

Remark 3.2. Using $\beta=1$ in (12) we have following recurrence relations for inverse moments of gos for Inverted Exponential distribution

$$
\mu_{r: n, m, k}^{-p}-\mu_{r-1: n, m, k}^{-p}=\frac{p}{\alpha \gamma_{r}}\left\{\mu_{r: n, m, k}^{-p+1}-\sum_{j=0}^{\infty} \frac{\alpha^{j}}{j !} \mu_{r: n, m, k}^{-p-j+1}\right\} .
$$

Remark 3.3. Using $m=0$ and $k=1$ in (12), following recurrence relation for inverse moments of order statistics for Inverted Weibull distribution is obtained

$$
\mu_{r: n}^{-p}-\mu_{r-1: n}^{-p}=\frac{p}{\alpha \beta(n+r-1)}\left\{\mu_{r: n}^{-p+\beta}-\sum_{j=0}^{\infty} \frac{\alpha^{j}}{j !} \mu_{r: n}^{-p-\beta(j-1)}\right\} .
$$

Remark 3.4. Using $m=-1$ in (12) we have following recurrence relation for inverse moments of $K$-record values for Inverted Weibull distribution

$$
\mu_{K(r)}^{-p}-\mu_{K(r-1)}^{-p}=\frac{p}{\alpha \beta k}\left\{\mu_{K(r)}^{-p+\beta}-\sum_{j=0}^{\infty} \frac{\alpha^{j}}{j !} \mu_{K(r)}^{-p-\beta(j-1)}\right\} .
$$


Remark 3.5. Recurrence relations for inverse moments of order statistics and record values for Inverted Exponential and Inverted Rayleigh distribution can be easily obtained from (15) and (16) by using $\beta=2$ and $\beta=1$ respectively.

\section{Relation for Product Moments}

Consider the following relation, given by Athar and Islam [4], for product moments of gos for any distribution $F(x)$

$$
\begin{aligned}
\mu_{r, s: n, m, k}^{p, q}-\mu_{r, s-1: n, m, k}^{p, q}= & \frac{q C_{s-2}}{(r-1) !(s-r-1) !} \int_{-\infty}^{\infty} \int_{x_{1}}^{\infty} x_{1}^{p} x_{2}^{q-1} \\
& \times f\left(x_{1}\right)\left\{1-F\left(x_{1}\right)\right\}^{m} g_{m}^{r-1}\left\{F\left(x_{1}\right)\right\} \\
& \times\left[h_{m}\left\{F\left(x_{2}\right)\right\}-h_{m}\left\{F\left(x_{1}\right)\right\}\right]^{s-r-1} \\
& \times\left\{1-F\left(x_{2}\right)\right\}^{\gamma_{s}} d x_{2} d x_{1},
\end{aligned}
$$

or

$$
\begin{aligned}
\mu_{r, s: n, m, k}^{p, q}-\mu_{r, s-1: n, m, k}^{p, q}= & \frac{q C_{s-2}}{(r-1) !(s-r-1) !} \int_{-\infty}^{\infty} \int_{x_{1}}^{\infty} x_{1}^{p} x_{2}^{q-1} \\
& \times f\left(x_{1}\right)\left\{1-F\left(x_{1}\right)\right\}^{m} g_{m}^{r-1}\left\{F\left(x_{1}\right)\right\} \\
& \times\left[h_{m}\left\{F\left(x_{2}\right)\right\}-h_{m}\left\{F\left(x_{1}\right)\right\}\right]^{s-r-1} \\
& \times\left\{1-F\left(x_{2}\right)\right\}^{\gamma_{s}-1}\left\{1-F\left(x_{2}\right)\right\} d x_{2} d x_{1} .
\end{aligned}
$$

Now using (7) in (19) we have following relation for product moments of gos for Inverted Weibull distribution under the assumption that $\beta>p$ and $\beta>q$.

$$
\begin{aligned}
\mu_{r, s: n, m, k}^{p, q}-\mu_{r, s-1: n, m, k}^{p, q}= & \frac{q C_{s-2}}{(r-1) !(s-r-1) !} \int_{0}^{\infty} \int_{x_{1}}^{\infty} x_{1}^{p} x_{2}^{q-1} \\
& \times f\left(x_{1}\right)\left\{1-F\left(x_{1}\right)\right\}^{m} g_{m}^{r-1}\left\{F\left(x_{1}\right)\right\} \\
& \times\left[h_{m}\left\{F\left(x_{2}\right)\right\}-h_{m}\left\{F\left(x_{1}\right)\right\}\right]^{s-r-1} \\
& \times\left\{1-F\left(x_{2}\right)\right\}^{\gamma_{s}-1} \\
& \times\left[\frac{x_{2}^{\beta+1}}{\alpha \beta}\left\{\exp \left(\frac{\alpha}{x_{2}^{\beta}}\right)-1\right\} f\left(x_{2}\right)\right] d x_{2} d x_{1} .
\end{aligned}
$$

or

$$
\begin{aligned}
\mu_{r, s: n, m, k}^{p, q}-\mu_{r, s-1: n, m, k}^{p, q}= & \frac{q}{\alpha \beta \gamma_{s}} \sum_{j=0}^{\infty} \frac{\alpha^{j}}{j !} \frac{C_{s-1}}{(r-1) !(s-r-1) !} \int_{0}^{\infty} \int_{x_{1}}^{\infty} x_{1}^{p} \\
& \times x_{2}^{q-\beta(j-1)} f\left(x_{1}\right) f\left(x_{2}\right)\left\{1-F\left(x_{1}\right)\right\}^{m} g_{m}^{r-1}\left\{F\left(x_{1}\right)\right\} \\
& \times\left[h_{m}\left\{F\left(x_{2}\right)\right\}-h_{m}\left\{F\left(x_{1}\right)\right\}\right]^{s-r-1} \\
& \times\left\{1-F\left(x_{2}\right)\right\}^{\gamma_{s}-1} d x_{2} d x_{1} \\
& -\frac{q}{\alpha \beta \gamma_{s}} \frac{C_{s-1}}{(r-1) !(s-r-1) !} \int_{0}^{\infty} \int_{x_{1}}^{\infty} x_{1}^{p} x_{2}^{q+\beta} \\
& \times f\left(x_{1}\right) f\left(x_{2}\right)\left\{1-F\left(x_{1}\right)\right\}^{m} g_{m}^{r-1}\left\{F\left(x_{1}\right)\right\} \\
& \times\left[h_{m}\left\{F\left(x_{2}\right)\right\}-h_{m}\left\{F\left(x_{1}\right)\right\}\right]^{s-r-1} \\
& \times\left\{1-F\left(x_{2}\right)\right\}^{\gamma_{s}-1} d x_{2} d x_{1}
\end{aligned}
$$


or

$$
\mu_{r, s: n, m, k}^{p, q}-\mu_{r, s-1: n, m, k}^{p, q}=\frac{q}{\alpha \beta \gamma_{s}}\left\{\sum_{j=0}^{\infty} \frac{\alpha^{j}}{j !} \mu_{r, s: n, m, k}^{p, q-\beta(j-1)}-\mu_{r, s: n, m, k}^{p, q+\beta}\right\}
$$

Remark 4.1. Using $m=0$ and $k=1$ in (18) we obtain recurrence relation for product moments of order statistics for Inverted Weibull distribution, for $\beta>p$ and $\beta>q$, as

$$
\mu_{r, s: n}^{p, q}-\mu_{r, s-1: n}^{p, q}=\frac{q}{\alpha(n+s-1)}\left\{\sum_{j=0}^{\infty} \frac{\alpha^{j}}{j !} \mu_{r, s: n}^{p, q-\beta(j-1)}-\mu_{r, s: n}^{p, q+\beta}\right\}
$$

Remark 4.2. Using $m=-1$ in (18) we obtain recurrence relation for product moments of $K-$ record values for Inverted Weibull distribution, for $\beta>p$ and $\beta>q$, as

$$
\mu_{K(r, s)}^{p, q}-\mu_{K(r, s-1)}^{p, q}=\frac{q}{k \alpha \beta}\left\{\sum_{j=0}^{\infty} \frac{\alpha^{j}}{j !} \mu_{K(r, s)}^{p, q-\beta(j-1)}-\mu_{K(r, s)}^{p, q+\beta}\right\}
$$

\section{Relation for Ratio Moments}

The ratio moments of gos are defined as

$$
\mu_{r, s: n, m, k}^{p,-q}=E\left(\frac{X_{r: n, m, k}^{p}}{X_{s: n, m, k}^{q}}\right)=\int_{-\infty}^{\infty} \int_{x_{1}}^{\infty} \frac{x_{1}^{p}}{x_{2}^{q}} f_{r, s: n, m, k}\left(x_{1}, x_{2}\right) d x_{2} d x_{1} .
$$

We now derive recurrence relation for ratio moments for inverse Weibull distribution. For this consider following relation for ratio moments of gos

$$
\begin{aligned}
\mu_{r, s: n, m, k}^{p,-q}-\mu_{r, s-1: n, m, k}^{p,-q}= & -\frac{q C_{s-2}}{(r-1) !(s-r-1) !} \int_{-\infty}^{\infty} \int_{x_{1}}^{\infty} x_{1}^{p} x_{2}^{-q-1} \\
& \times f\left(x_{1}\right)\left\{1-F\left(x_{1}\right)\right\}^{m} g_{m}^{r-1}\left\{F\left(x_{1}\right)\right\} \\
& \times\left[h_{m}\left\{F\left(x_{2}\right)\right\}-h_{m}\left\{F\left(x_{1}\right)\right\}\right]^{s-r-1} \\
& \times\left\{1-F\left(x_{2}\right)\right\}^{\gamma_{s}} d x_{2} d x_{1},
\end{aligned}
$$

or

$$
\begin{aligned}
\mu_{r, s: n, m, k}^{p,-q}-\mu_{r, s-1: n, m, k}^{p,-q}= & -\frac{q C_{s-2}}{(r-1) !(s-r-1) !} \int_{-\infty}^{\infty} \int_{x_{1}}^{\infty} x_{1}^{p} x_{2}^{-q-1} \\
& \times f\left(x_{1}\right)\left\{1-F\left(x_{1}\right)\right\}^{m} g_{m}^{r-1}\left\{F\left(x_{1}\right)\right\} \\
& \times\left[h_{m}\left\{F\left(x_{2}\right)\right\}-h_{m}\left\{F\left(x_{1}\right)\right\}\right]^{s-r-1} \\
& \times\left\{1-F\left(x_{2}\right)\right\}^{\gamma_{s}-1}\left\{1-F\left(x_{2}\right)\right\} d x_{2} d x_{1} .
\end{aligned}
$$


Now using (7) in (21) we have following relation for ratio moments of gos for Inverted Weibull distribution when $\beta>p, q>\beta$ and $p-q>\beta$.

$$
\begin{aligned}
\mu_{r, s: n, m, k}^{p,-q}-\mu_{r, s-1: n, m, k}^{p,-q}= & -\frac{q C_{s-2}}{(r-1) !(s-r-1) !} \int_{0}^{\infty} \int_{x_{1}}^{\infty} x_{1}^{p} x_{2}^{-q-1} \\
& \times f\left(x_{1}\right)\left\{1-F\left(x_{1}\right)\right\}^{m} g_{m}^{r-1}\left\{F\left(x_{1}\right)\right\} \\
& \times\left[h_{m}\left\{F\left(x_{2}\right)\right\}-h_{m}\left\{F\left(x_{1}\right)\right\}\right]^{s-r-1} \\
& \times\left\{1-F\left(x_{2}\right)\right\}^{\gamma_{s}-1} \\
& \times\left[\frac{x_{2}^{\beta+1}}{\alpha \beta}\left\{\exp \left(\frac{\alpha}{x_{2}^{\beta}}\right)-1\right\} f\left(x_{2}\right)\right] d x_{2} d x_{1} .
\end{aligned}
$$

or

$$
\begin{aligned}
\mu_{r, s: n, m, k}^{p,-q}-\mu_{r, s-1: n, m, k}^{p,-q}= & -\frac{q}{\alpha \beta \gamma_{s}} \sum_{j=0}^{\infty} \frac{\alpha^{j}}{j !} \frac{C_{s-1}}{(r-1) !(s-r-1) !} \int_{0}^{\infty} \int_{x_{1}}^{\infty} x_{1}^{p} \\
& \times x_{2}^{-q-\beta(j-1)} f\left(x_{1}\right) f\left(x_{2}\right)\left\{1-F\left(x_{1}\right)\right\}^{m} g_{m}^{r-1}\left\{F\left(x_{1}\right)\right\} \\
& \times\left[h_{m}\left\{F\left(x_{2}\right)\right\}-h_{m}\left\{F\left(x_{1}\right)\right\}\right]^{s-r-1} \\
& \times\left\{1-F\left(x_{2}\right)\right\}^{\gamma_{s}-1} d x_{2} d x_{1} \\
& +\frac{q}{\alpha \beta \gamma_{s}} \frac{C_{s-1}}{(r-1) !(s-r-1) !} \int_{0}^{\infty} \int_{x_{1}}^{\infty} x_{1}^{p} x_{2}^{-q+\beta} \\
& \times f\left(x_{1}\right) f\left(x_{2}\right)\left\{1-F\left(x_{1}\right)\right\}^{m} g_{m}^{r-1}\left\{F\left(x_{1}\right)\right\} \\
& \times\left[h_{m}\left\{F\left(x_{2}\right)\right\}-h_{m}\left\{F\left(x_{1}\right)\right\}\right]^{s-r-1} \\
& \times\left\{1-F\left(x_{2}\right)\right\}^{\gamma_{s}-1} d x_{2} d x_{1}
\end{aligned}
$$

or

$$
\mu_{r, s: n, m, k}^{p,-q}-\mu_{r, s-1: n, m, k}^{p,-q}=\frac{q}{\alpha \beta \gamma_{s}}\left\{\mu_{r, s: n, m, k}^{p,-q+\beta}-\sum_{j=0}^{\infty} \frac{\alpha^{j}}{j !} \mu_{r, s: n, m, k}^{p, q-\beta(j-1)}\right\} .
$$

Remark 5.1. Using $m=0$ and $k=1$ in (22) we have following recurrence relation for ratio moments of order statistics for Inverted Weibull distribution as

$$
\mu_{r, s: n}^{p,-q}-\mu_{r, s-1: n}^{p,-q}=\frac{q}{\alpha(n+s-1)}\left\{\mu_{r, s: n}^{p,-q+\beta}-\sum_{j=0}^{\infty} \frac{\alpha^{j}}{j !} \mu_{r, s: n}^{p,-q-\beta(j-1)}\right\} .
$$

Remark 5.2. Using $m=-1$ in (22) we obtain following recurrence relation for ratio moments of record values for Inverted Weibull distribution as

$$
\mu_{K(r, s)}^{p,-q}-\mu_{K(r, s-1)}^{p,-q}=\frac{q}{k \alpha \beta}\left\{\mu_{K(r, s)}^{p,-q+\beta}-\sum_{j=0}^{\infty} \frac{\alpha^{j}}{j !} \mu_{K(r, s)}^{p,-q-\beta(j-1)}\right\} .
$$

\section{Some Characterizations}

In this section we present some characterizations of the Inverse Weibull distribution based upon the conditional moments. In order to give main theorems we first give some lemma.

Lemma 6.1. If $X$ is an absolutely continuous random variable with cumulative distribution function $F(x)$ and probability density function $f(x)$ with $a=\sup (x \mid F(x)>0)$ and $b=\inf (x \mid F(x)<$ 
1).We assume $E(X)$ exists. If for a given $x, a<x<b$,

$$
E(X \mid X<x)=g(x) \tau(x)
$$

where $g(x)$ is a differentiable function in $a, x<b$, and $\tau(x)=f(x) / F(x)$, then

$$
f(x)=c \exp \left\{\int\left(\frac{x-g^{\prime}(x)}{g(x)}\right) d x\right\},
$$

where $c$ is determined by the condition $\frac{1}{c}=\int_{a}^{b} f(x) d x$.

Proof. Consider

$$
g(x)=\frac{1}{f(x)} \int_{0}^{x} u f(u) d u
$$

then

$$
g(x) f(x)=\int_{0}^{x} u f(u) d u .
$$

Differentiating both sides we have

$$
x f(x)=g^{\prime}(x) f(x)+g(x) f^{\prime}(x),
$$

which on simplification becomes

$$
\frac{f^{\prime}(x)}{f(x)}=\frac{x-g^{\prime}(x)}{g(x)}
$$

Integrating both sides with respect to $x$ we have

$$
f(x)=c \exp \left\{\int\left(\frac{x-g^{\prime}(x)}{g(x)}\right) d x\right\},
$$

where $c$ is determined such that $\frac{1}{c}=1$.

Lemma 6.2. Suppose that $X$ is an absolutely continuous random variable with cdf $F(x)$ with $F(0)=0$ and $F(x)>0$ for all $x>0$. We assume that the pdf of $X$ as $f(x)$ and $f^{\prime}(x)$ exists for all $x>0$. For a continuous function $g(x)$ on $0<x<\infty$ with finite $E\{g(x)\}$ such that

$$
E\{g(X) \mid X \geq x\}=h(x) r(x)
$$

where $h(x)$ is a differential function in $x>0$ and $r(x)=f(x) /\{1-F(x)\}$, then

$$
f(x)=c \exp \left\{-\int\left(\frac{g(x)+h^{\prime}(x)}{h(x)}\right)\right\} d x
$$

and $c$ is determined by the condition $\int_{0}^{\infty} f(x) d x=1$. 
Proof. Consider

$$
\int_{x}^{\infty} g(u) f(u) d u=f(x) h(x)
$$

Differentiating the above expression, we obtain

$$
-g(x) f(x)=f(x) h^{\prime}(x)+f^{\prime}(x) h(x) .
$$

Simplifying, we have

$$
\frac{f^{\prime}(x)}{f(x)}=-\frac{g(x)+h^{\prime}(x)}{h(x)}
$$

Integrating both sides of the above equation we obtain (26) and $\mathrm{c}$ is determined by the condition $\int_{0}^{\infty} f(x) d x=1$.

We now give the main theorems.

Theorem 6.1. If $X$ is an absolutely continuous positive random variable with cumulative distribution function $G(x)$ and probability density function $g(x)$ such that $E(X)$ exists. Then $E(X \mid X \leq x)=$ $h(x) \tau(x)$, where $\tau(x)=g(x) / G(x)$

$$
h(x)=\frac{x^{\beta+2}}{\alpha \beta}-\frac{x^{\beta+1}}{\alpha^{1-\frac{1}{\beta}} \beta^{2}} x^{\beta+1} e^{-\frac{\alpha}{x^{\beta}}} \Gamma_{\frac{\alpha}{x^{\beta}}}\left(-\frac{1}{\beta}\right)
$$

and

$$
\Gamma_{x}(\alpha)=\int_{x}^{\infty} u^{\alpha-1} e^{-u} d u, \alpha>0, \beta>1
$$

holds if and only if

$$
g(x)=\frac{\alpha \beta}{x^{\beta+1}} \exp \left(-\frac{\alpha}{x^{\beta}}\right), x>0, \alpha>0 \text { and } \beta>0 .
$$

Proof. We first prove the necessity part. For this consider

$$
g(x)=\frac{\alpha \beta}{x^{\beta+1}} \exp \left(-\frac{\alpha}{x^{\beta}}\right),
$$

then

$$
\begin{aligned}
h(x) & =\frac{1}{g(x)} \int_{0}^{x} u f(u) d u=\frac{x^{\beta+2}}{\alpha \beta}-\frac{x^{\beta+1}}{\alpha \beta} e^{\frac{\alpha}{x^{\beta}}} \int_{0}^{x} e^{-\frac{\alpha}{u^{\beta}}} d u \\
& =\frac{x^{\beta+2}}{\alpha \beta}-\frac{x^{\beta+1}}{\alpha^{1-\frac{1}{\beta}} \beta^{2}} e^{\frac{\alpha}{x^{\beta}}} \Gamma_{\frac{\alpha}{x^{\beta}}}\left(-\frac{1}{\beta}\right),
\end{aligned}
$$

which is (27). 
Now for sufficient condition we need to prove that (27) implies (4). For this consider (27) as

$$
h(x)=\frac{x^{\beta+2}}{\alpha \beta}-\frac{x^{\beta+1}}{\alpha^{1-\frac{1}{\beta}} \beta^{2}} e^{\alpha x^{-\beta}} \Gamma_{\frac{\alpha}{x^{\beta}}}\left(-\frac{1}{\beta}\right) .
$$

Differentiating above equation with respect to $x$ we have

$$
\begin{aligned}
h^{\prime}(x) & =\frac{\beta+2}{\alpha \beta} x^{\beta+1}-\frac{x^{\beta+1}}{\alpha \beta}-\Gamma_{\frac{\alpha}{x^{\beta}}}\left(-\frac{1}{\beta}\right) \frac{d}{d x}\left(\frac{x^{\beta+1}}{\alpha^{1-\frac{1}{\beta}} \beta^{2}} e^{\alpha x^{-\beta}}\right) \\
& =\frac{\beta+1}{\alpha \beta} x^{\beta+1}-\Gamma_{\frac{\alpha}{x^{\beta}}}\left(-\frac{1}{\beta}\right)\left\{\frac{x^{\beta+1}}{\alpha^{1-\frac{1}{\beta}} \beta^{2}} e^{\alpha x^{-\beta}}\right\}\left\{\frac{\beta+1}{x}-\frac{\alpha \beta}{x^{\beta+1}}\right\} \\
& =x-\left(\frac{\alpha \beta}{x^{\beta+1}}-\frac{\beta+1}{x}\right)\left\{\frac{x^{\beta+2}}{\alpha \beta}-\frac{x^{\beta+1}}{\alpha^{1-\frac{1}{\beta}} \beta^{2}} e^{\alpha x^{-\beta}} \Gamma_{\frac{\alpha}{x^{\beta}}}\left(-\frac{1}{\beta}\right)\right\}
\end{aligned}
$$

or

$$
h \prime(x)=x-\left(\frac{\alpha \beta}{x^{\beta+1}}-\frac{\beta+1}{x}\right) h(x) .
$$

Thus we have

$$
\frac{x-h \prime(x)}{h(x)}=\left(\frac{\alpha \beta}{x^{\beta+1}}-\frac{\beta+1}{x}\right) .
$$

Hence using the Lemma we have

$$
\frac{g^{\prime}(x)}{g(x)}=\left(\frac{\alpha \beta}{x^{\beta+1}}-\frac{\beta+1}{x}\right) .
$$

Integrating we have

$$
g(x)=\frac{c}{x^{\beta+1}} e^{-\frac{\alpha}{x^{\beta}}},
$$

where $c$ is determined by using

$$
\frac{1}{c}=\int_{0}^{\infty} \frac{c}{x^{\beta+1}} e^{\alpha x^{-\beta}} d x=\frac{1}{\alpha \beta},
$$

hence

$$
g(x)=\frac{\alpha \beta}{x^{\beta+1}} \exp \left(-\frac{\alpha}{x^{\beta}}\right),
$$

as asserted.

Theorem 6.2. Suppose that $X$ is an absolutely continuous random variable with $c d f G(x)$ such that $G(0)=0$ and $G(x)>0$ for all $x>0$. We assume that the $p d f$ of $X$ and $g(x)$ and $g^{\prime}(x)$ exists for all $x>0$ and $E(X)$ also exists. Then $E(X \mid X \geq x)=h_{0}(x) \tau(x)$, where $\tau(x)=g(x) /[1-G(x)]$,

$$
h_{0}(x)=\frac{\alpha^{1+\frac{1}{\beta}}}{\beta^{2}} \exp \left(\frac{\alpha}{x^{\beta}}\right) \Gamma\left(\frac{\alpha}{x^{\beta}}, 1\right), \beta>1
$$

and $\Gamma(x, n)=\int_{0}^{x} u^{n-1} e^{-u} d u$, holds if and only if 


$$
g(x)=\frac{\alpha \beta}{x^{\beta+1}} \exp \left(-\frac{\alpha}{x^{\beta}}\right), x \geq 0, \alpha>0 \text { and } \beta>1 .
$$

Proof. We have

$$
g(x) h_{0}(x)=\int_{x}^{\infty} u \frac{\alpha \beta}{u^{\beta+1}} \exp \left(-\frac{\alpha}{u^{\beta}}\right) d u=\alpha^{1+\frac{1}{\beta}} \Gamma\left(\frac{\alpha}{x^{\beta}}, 1-\frac{1}{\beta}\right), \beta>1 .
$$

Thus

$$
h_{0}(x)=\frac{\alpha^{1+\frac{1}{\beta}}}{\beta} \exp \left(\frac{\alpha}{x^{\beta}}\right) \Gamma\left(\frac{\alpha}{x^{\beta}}, 1-\frac{1}{\beta}\right) .
$$

Now

$$
\begin{aligned}
h_{0}^{\prime}(x) & =-x-\frac{\alpha^{1+\frac{1}{\beta}} x^{1+\beta}}{\beta} \exp \left(\frac{\alpha}{x^{\beta}}\right) \Gamma\left(\frac{\alpha}{x^{\beta}}, 1-\frac{1}{\beta}\right)\left(-\frac{1+\beta}{x}+\frac{\alpha \beta}{x^{1+\beta}}\right) \\
& =-x-h_{0}(x)\left(-\frac{1+\beta}{x}-\frac{\alpha \beta}{x^{1+\beta}}\right) .
\end{aligned}
$$

Thus

$$
-\frac{x+h_{0}^{\prime}(x)}{h_{0}(x)}=-\frac{1+\beta}{x}+\frac{\alpha \beta}{x^{1+\beta}}
$$

By Lemma 3, we have

$$
\frac{g^{\prime}(x)}{g(x)}=-\frac{1+\beta}{x}+\frac{\alpha \beta}{x^{1+\beta}}
$$

Integrating both sides of the above equation with respect to $x$, we obtain

$$
g(x)=c x^{1+\beta} \exp \left(-\frac{\alpha}{x^{\beta}}\right)
$$

where $c$ is a constant and is determined by using the condition $\int_{0}^{\infty} g(x) d x=1$. Using the value of $c$ we have

$$
g(x)=\frac{\alpha \beta}{x^{\beta+1}} \exp \left(-\frac{\alpha}{x^{\beta}}\right), x \geq 0, \alpha>0 \text { and } \beta>1,
$$

as asserted.

\section{Conclusion}

In this paper we have presented the recurrence relations for single, inverse, product and ratio moments of gos when sample is available from Inverse Weibull distribution alongside a characterization of the distribution in terms of conditional moments. These relations provide corresponding relations for single and product moments of order and record statistics as special case. The relations have been used to obtain recurrence relations for single and product moments of gos for Inverse Exponential and Inverse Rayleigh distribution. 


\section{References}

[1] Arnold, B. C., Balakrishnan, N. and Nagaraja, H. N. (2008). A First Course in Order Statistics, SIAM, USA.

[2] Ahsanullah, M. (1991). On Record Values from the Generalized Pareto Distribution. Pak. J. Stat., 7 (2), 129-136.

[3] Ahsanullah, M. (1995) Record Statistics, Nova Science Publisher, New York.

[4] Athar, H. and Islam, H. M. (2004). Recurrence relations between single and product moments of generalized order statistics from a general class of distributions. Metron, LXII, 327-337.

[5] Athar, H., Nayabuddin and Khwaja, S. K. (2012). Relations for moments of generalized order statistics from Marshall-Olkin extended Weibull distribution and its characterization, ProbStat Forum, 5, 127 132.

[6] Balakrishnan, N., and Ahsanullah, M. (1995). Relations for Single and Product Moments of Record Values from Exponential Distribution. J. Apl. Statist. Sci., 2 (1), 73-88.

[7] Balakrishnan, N., Ahsanullah, M. and Chan, P. S. (1992). Relations for Single and Product Moments of Record Values from Gumbel Distribution. Stat. and Prob. Letters, 15, 223-227.

[8] Burkschat, M., Cramer, E. and Kamps, U. (2003). Dual generalized order statistics. Metron, 61(1), 13-26.

[9] Bieniek, M. and Szynal, D. (2002). Recurrence relations for distribution functions and moments of kth record values, J. Math.Sci. 111(3), 3511-3519.

[10] Chandler, K. N. (1952). The distribution and frequency of record values, J. Royal Statist. Soc. B, 14, 220-228.

[11] Cole, R. H. (1951). Relations between moments of order statistics, Ann. Math. Statist., 22, 308-310.

[12] David, H. A. and Nagaraja, H. N. (2003). Order Statistics, John Wiley Inc., USA.

[13] Kamps, U. (1995). A concept of generalized order statistics, J. Statist. Plann. Inference, 48, 1-23.

[14] Kotb, M. S., El-Din, M. M. M. and Newer, H. A. (2013). Recurrence relations for single and product moments of lower generalized order statistics from a general class of distributions and its characterizations, Inter. J. of Pure and Applied Maths., 87(2),229-247.

[15] Kumar, D. (2011). Generalized Order Statistics from Kumaraswamy Distribution and its Characterization, Tamsui Oxford J. of Info. Math. Sci., 27(4), 463-476.

[16] Mohsin, M., Shahbaz, M. Q. and Kibria, G. (2010) Recurrence Relations for moments of Generalized Order Statistics for Rayleigh distribution, App. Math. \& Info. Sci. Vol. 4(3), 273-279

[17] Pawlas, P., and Szynal, D. (2000). Recurrence relations for single and product moment of k-th record values from Weibull distribution and a characterization, Journal of Applied Statistical Science, 10, 1725.

[18] Pawlas, P., and Szynal, D. (2001). Recurrence relations for single and product moment of lower generalized order statistics from Inverse Weibull distribution, Demonstratio Mathematica, 34(2), 353-358. 